\title{
CONVERGENCE ANALYSIS OF THE GAUSS-SEIDEL PRECONDITIONER FOR DISCRETIZED ONE DIMENSIONAL EULER EQUATIONS*
}

\author{
ARNOLD REUSKEN ${ }^{\dagger}$
}

\begin{abstract}
We consider the nonlinear system of equations that results from the Van Leer flux vector-splitting discretization of the one dimensional Euler equations. This nonlinear system is linearized at the discrete solution. The main topic of this paper is a convergence analysis of blockGauss-Seidel methods applied to this linear system of equations. Both the lexicographic and the symmetric block-Gauss-Seidel method are considered. We derive results which quantify the quality of these methods as preconditioners. These results show, for example, that for the subsonic case the symmetric Gauss-Seidel method can be expected to be a much better preconditioner than the lexicographic variant. Sharp bounds for the condition number of the preconditioned matrix are derived.
\end{abstract}

AMS subject classifications. 65F10, 65N22, 65N06

Key words. Gauss-Seidel method, Euler equations, convergence analysis

DOI. $10.1137 /$ S0036142902407393

1. Introduction. In this paper we consider iterative methods for discrete stationary Euler equations. Two important solution approaches known from the literature are the following. First, one can use some "simple" explicit iterative method, like a block nonlinear Gauss-Seidel method or a Runge-Kutta method (obtained by introducing an artificial time variable), which then is accelerated by multigrid techniques (e.g., $[12,13,16,20,25,27])$. The second approach is based on linearization combined with fast iterative solvers for large sparse linear systems, such as multigrid solvers or (preconditioned) Krylov-subspace methods. A typical example of this is the Newton-Krylov technique from $[5,14,15,18,19,24]$. In the literature one can find many studies in which different iterative solution techniques for solving stationary (or instationary) discrete Euler equations are compared (e.g., [17, 26]). There are, however, as far as we know, no rigorous theoretical results available which yield any insight into convergence properties of certain iterative methods applied to (linearized) discrete Euler equations. In this paper a first step towards such theoretical results is made.

In this paper, as a model problem we consider the stationary Euler equations that model one dimensional subsonic and transonic flows through a nozzle [11, 21], and use the Van Leer flux vector-splitting method for discretization. The discrete nonlinear problem is linearized at the discrete solution. We apply a GMRES method with block-Gauss-Seidel preconditioning to this Jacobian linear problem. In the Gauss-Seidel preconditioner the three unknowns at each grid point are collected in a block and updated simultaneously. (This is also often called a collective GaussSeidel method.) Both a lexicographic (LGS) and a symmetric (SGS) Gauss-Seidel

* Received by the editors May 10, 2002, accepted for publication (in revised form) February 26, 2003; published electronically September 9, 2003. This work was supported by the Deutsche Forschungsgemeinschaft in the Collaborative Research Centre SFB 401 "Flow Modulation and FluidStructure Interaction at Airplane Wings" of the RWTH Aachen, University of Technology, Aachen, Germany.

http://www.siam.org/journals/sinum/41-4/40739.html

${ }^{\dagger}$ Institut für Geometrie und Praktische Mathematik, Rheinisch-Westfälische Technische Hochschule Aachen, D-52056 Aachen, Germany (reusken@igpm.rwth-aachen.de). 
method are used. We emphasize that we do not recommend using such an iterative method for these one dimensional linearized Euler equations, because the Jacobian matrix has a block-tridiagonal structure with $3 \times 3$ blocks, and thus a direct solver is efficient for this problem. Our main interest, however, is not the efficient solution of these one dimensional Euler equations, but a better understanding of convergence properties of the block-Gauss-Seidel method applied to discrete Euler equations.

As is well known, direction of flow essentially influences not only the discretization of Euler equations, but also the convergence of iterative methods. If the flow is subsonic, then even in the one dimensional case the LGS method cannot be consistent with the flow direction. In one dimensional flow one has only two directions, and thus the SGS method can be expected to be a fast iterative solver. These elementary observations are part of common knowledge. However, even for one dimensional flows many questions related to Gauss-Seidel preconditioning are still unanswered. As will be illustrated by numerical experiments, for GMRES with block-Gauss-Seidel preconditioning there are some interesting dependencies of the rate of convergence on the Mach number and the mesh size. As far as we know, there is no analysis available which explains these dependencies. The main topic of the paper is a theoretical analysis in which we try to explain some of the convergence phenomena that are observed in the numerical experiments. In this analysis we use the technique of "frozen coefficients"; i.e., we linearize the discrete Euler equations at a function triple $(\rho, u, p)$ (density, velocity, pressure) which is constant as a function of the space variable and is such that the solution is subsonic. We consider the LGS and SGS methods applied to this problem and derive results which quantify the quality of these methods as a preconditioner. Our results show, for example, that the SGS method can be expected to be a (much) better preconditioner than the LGS method. Sharp bounds for the condition number of the preconditioned matrix are derived, which show that in case of the SGS preconditioner for a large range of Mach numbers $M \in\left(M_{0}, 1\right)$ this condition number increases only (very) slowly if the grid size decreases.

We realize that although some first theoretical results are given in this paper, we are still far from a complete theoretical convergence analysis of Gauss-Seidel methods applied to linearized discrete one dimensional Euler equations. The theoretical analysis presented supports the numerical observation that for many subsonic and transonic one dimensional linearized Euler equations the SGS method is a (very) effective preconditioner. However, as already noted above, in the one dimensional case a direct solver is the best choice. In two and three dimensional problems, however, block-Gauss-Seidel techniques or other basic iterative methods (ILU) combined with Krylov subspace methods can result in very efficient solvers [3, 4, 6, 19]. Clearly, in higher dimensions flow has many directions to go and the relation between a GaussSeidel-type splitting and direction of flow becomes much more complicated. This then makes the analysis of this class of iterative methods much more difficult, as in the one dimensional case. We do not claim that our analysis can easily be applied to the much more interesting higher dimensional case. Nevertheless, starting from the results presented in this paper we do see some possibilities for the analysis of a two dimensional problem. These are briefly discussed in Remark 3 at the end of the paper.

2. The one dimensional nozzle flow and its discretization. We consider the stationary quasi-one dimensional Euler flow in a channel of varying cross section 
$S(x)(x \in \mathbb{R})$. This problem can be modeled by the equations (cf. [11, 21])

$$
\left\{\begin{array}{l}
\frac{d(\rho u S)}{d x}=0, \\
\frac{d\left(\rho u^{2} S+p S\right)}{d x}=p \frac{d S}{d x}, \\
\frac{d(\rho u H S)}{d x}=0
\end{array}\right.
$$

with density $\rho$, velocity $u$, pressure $p$, and stagnation enthalpy $H=E+\frac{p}{\rho}$. Further relations are

$$
E=e+\frac{1}{2} u^{2}, \quad p=(\gamma-1) \rho e .
$$

Here $e$ denotes the internal energy and $\gamma$ is a gas parameter (ratio of specific heats; $\gamma=1.4$ for air). As unknowns one can take the primitive variables $V:=(\rho, u, p)^{T}$. We introduce the conservative variables $U$, the source term $Q_{S}$, and the flux function $f$ :

$$
\begin{aligned}
& U=\left(\begin{array}{l}
u_{1} \\
u_{2} \\
u_{3}
\end{array}\right):=S\left(\begin{array}{c}
\rho \\
\rho u \\
\rho E
\end{array}\right), \quad Q_{S}(U):=\left(\begin{array}{c}
0 \\
\frac{d S}{d x} \\
0
\end{array}\right)=\left(\begin{array}{c}
0 \\
\left.(\gamma-1)\left(u_{3}-\frac{1}{2} \frac{u_{2}^{2}}{u_{1}}\right) \frac{d \ln S}{d x}\right) \\
0
\end{array}\right) \\
& f(U):=S\left(\begin{array}{c}
\rho u \\
\rho u^{2}+p \\
\rho u H
\end{array}\right)=\left(\begin{array}{c}
u_{2} \\
\frac{1}{2}(3-\gamma) \frac{u_{2}^{2}}{u_{1}}+(\gamma-1) u_{3} \\
\gamma \frac{u_{2} u_{3}}{u_{1}}-\frac{1}{2}(\gamma-1) \frac{u_{2}^{3}}{u_{1}^{2}}
\end{array}\right) .
\end{aligned}
$$

In compact form the problem can be represented as

$$
f(U)_{x}=Q_{S}(U) .
$$

Note that for $S(x) \equiv 1$ we obtain the homogeneous one dimensional Euler equations. Formulas for the transformation between the primitive variables $V$ and the conservative variables $U$ are known (cf. [11]). Important quantities are the speed of sound $c=\left(\gamma p \rho^{-1}\right)^{\frac{1}{2}}$ and the Mach number $M=u c^{-1}$. In our experiments we take the following nozzle with throat at $x=1$ :

$$
S(x)= \begin{cases}1+1 \frac{1}{2}\left(1-\frac{1}{5}(x+4)\right)^{2} & \text { for } 0 \leq x \leq 1, \\ 1+\frac{1}{2}\left(1-\frac{1}{5}(x+4)\right)^{2} & \text { for } 1 \leq x \leq 4 .\end{cases}
$$

Nozzle flows are well-known test cases for steady-state computations (cf. [11, 13]). By specifying certain problem parameters (inflow Mach number and critical throat section), the problem (2.1) can have several types of solutions: a smooth subsonic flow, a smooth hypersonic flow, a transonic flow without shocks, or a transonic flow with shocks. Moreover, these solutions depend on only one parameter (for example, the Mach number $M=M(x)$ ), and a simple procedure for computing the exact solution of the continuous problem is available (cf. [11, section 16.6.4]). For two cases the function $x \rightarrow M(x)$ corresponding to the exact solution of the problem (2.1), (2.2) is shown in Figures 2.1 and 2.2. In Figure 2.1 we have a smooth subsonic flow with critical throat section $S^{*}=0.5$. The solution in Figure 2.2 corresponds to a transonic flow with a critical throat value $S^{*}=1$, which equals the throat value $S(1)$, and a shock at $x=3$. 


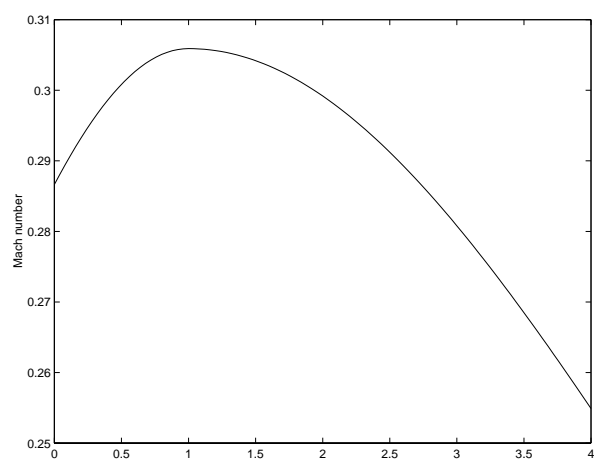

FIG. 2.1. $x \rightarrow M(x)$ for a smooth subsonic flow.

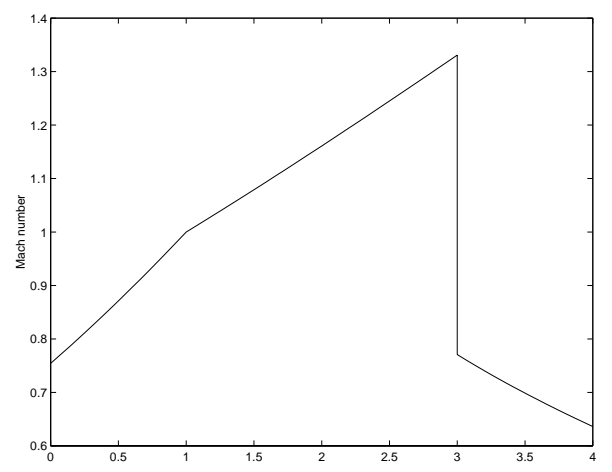

Fig. 2.2. $x \rightarrow M(x)$ for a transonic flow with shock.

We now outline the numerical solution method for this test problem (for which the exact solution is available). We consider only problems with subsonic inflow and outflow conditions $(0<M(0)<1$ and $0<M(4)<1)$. For the boundary conditions we prescribe values for $\rho$ and $u$ at the inflow boundary $x=0$, and for $p$ at the outflow boundary $x=4$. We use a uniform grid $x_{i}=i h, 0 \leq i \leq n+1$, with a mesh size $h=4 /(n+1)$. We introduce the discrete unknowns

$$
U_{i}:=\left(\begin{array}{l}
u_{1}\left(x_{i}\right) \\
u_{2}\left(x_{i}\right) \\
u_{3}\left(x_{i}\right)
\end{array}\right), \quad \mathbf{U}:=\left(U_{i}\right)_{0 \leq i \leq n+1} .
$$

For the discretization at the boundaries we use compatibility relations as discussed in [11, section 19.1.2]; i.e., at the inflow boundary we discretize with one-sided differences the equation $(u-c)\left(\frac{d u}{d x}-\frac{1}{\rho c} \frac{d p}{d x}\right)=u c \frac{d \ln S}{d x}$ that corresponds to the left-going characteristic. Similarly, the two right-going characteristic equations at $x=4$ are discretized using one-sided differences. Together with the prescribed boundary values this yields equations

$$
\begin{aligned}
F_{0}: \mathbb{R}^{6} \rightarrow \mathbb{R}^{3}, & F_{0}\left(U_{0}, U_{1}\right)=0, \\
F_{n+1}: & \mathbb{R}^{6} \rightarrow \mathbb{R}^{3}, \quad F_{n+1}\left(U_{n}, U_{n+1}\right)=0 .
\end{aligned}
$$

For the discretization in the interior grid points we use an upwind method based on the Van Leer flux vector-splitting (see [11, 28]):

$$
\begin{aligned}
f(V) & =f^{+}(V)+f^{-}(V), \\
f^{+}(V) & :=\frac{\rho}{4 c}(u+c)^{2}\left(\begin{array}{c}
1 \\
\frac{(\gamma-1) u+2 c}{\gamma} \\
\frac{((\gamma-1) u+2 c)^{2}}{2\left(\gamma^{2}-1\right)}
\end{array}\right) \quad \text { if } \quad-1 \leq M \leq 1, \\
f^{+} & :=0 \quad \text { if } M \leq-1, \quad f^{+}:=f \quad \text { if } \quad M \geq 1 .
\end{aligned}
$$

We use backward differences for the approximation of $f^{+}(U)_{x}$, and forward differences for the approximation of $f^{-}(U)_{x}$. This yields the equations

$$
F_{i}\left(U_{i-1}, U_{i}, U_{i+1}\right):=-f^{+}\left(U_{i-1}\right)+f^{+}\left(U_{i}\right)-f^{-}\left(U_{i}\right)+f^{-}\left(U_{i+1}\right)-h Q_{S}\left(U_{i}\right)=0
$$


for $i=1, \ldots, n$. The equations (2.3), (2.4), and (2.6) yield a nonlinear system of equations

$$
F: \mathbb{R}^{3(n+2)} \rightarrow \mathbb{R}^{3(n+2)}, \quad F(\mathbf{U})=0 .
$$

For the iterative solution of this problem we apply the Newton method. The Jacobian matrices $D F(\mathbf{U}) \in \mathbb{R}^{3(n+2) \times 3(n+2)}$ have a block-tridiagonal structure. Hence, the linear systems in the Newton iteration can be solved efficiently using a direct method. The main topic of this paper is the analysis of block-Gauss-Seidel iterative methods applied to these linear systems. We emphasize that we do not suggest using such a Gauss-Seidel method as an efficient solver in this one dimensional setting. The analysis for the one dimensional case is a first step towards a better theoretical understanding of basic iterative methods applied to two or three dimensional linearized Euler equations.

3. Numerical experiments. In this section we show results of a few numerical experiments which illustrate some interesting phenomena related to the rate of convergence of block-Gauss-Seidel methods. Let $\mathbf{U}_{h}^{*}$ be the solution of the discrete problem (2.7). We consider the linear system

$$
D F\left(\mathbf{U}_{h}^{*}\right) \mathbf{v}=\mathbf{b} .
$$

In the experiments we take $\mathbf{b}=(1, \ldots, 1)^{T}$, and for the starting vector in the iterative method we use $\mathbf{v}^{0}=0$. It turns out that in many cases (often due to the treatment of the boundary conditions) the block-Gauss-Seidel method does not converge. It turns out, however, that the method is a (very) good preconditioner. Hence, we use the block-Gauss-Seidel method in combination with a Krylov subspace method. We choose the GMRES $(m)$ iterative method. Experiments with BiCGSTAB yielded similar results.

We use the LGS and SGS methods. In the GMRES method we make a restart after $m=20$ iterations. The choice $m=20$ is rather arbitrary, however; for other values of $m \in[10,40]$ we observe similar phenomena. We use the GMRES $(m)$ implementation in MATLAB. In a first experiment, as a comparison for other results, we consider a standard very simple model problem. We take the one dimensional diffusion equation $-u_{x x}=g$ discretized by second order differences. This results in an $n \times n$ tridiagonal matrix tridiag $(-1,2,-1)$. For different $n$-values the convergence history of the SGS-GMRES(20) iterative solver applied to this problem is shown in Figure 3.1. For the linearized compressible Euler equations (3.1) we show results for the following problems.

Problem 1. We consider a problem with a smooth subsonic solution, as shown in Figure 2.1. The convergence history of the SGS-GMRES(20) method is shown in Figure 3.2 .

Problem 2. We take a smooth subsonic flow with larger Mach numbers than in Problem 1. The solution is shown in Figure 3.3 (with critical throat value $S^{*}=0.85$ ). The corresponding convergence history is presented in Figure 3.4.

Problem 3. We consider a transonic flow with a shock, as shown in Figure 2.2. The convergence behavior of the SGS-GMRES(20) solver is shown in Figure 3.5. If instead of SGS we use the LGS preconditioner, we obtain the results in Figure 3.6.

From these experiments we observe that in all three problems the rate of convergence of the SGS-GMRES(20) method is (much) higher than for the one dimensional discrete Poisson equation. We also see that in Problem 2 (subsonic flow with relatively high Mach numbers) the rate of convergence is much higher than in Problem 


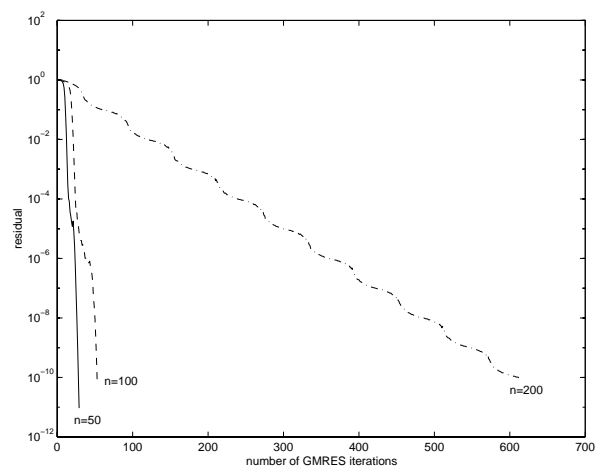

Fig. 3.1. SGS-GMRES(20) method applied to a one dimensional Poisson equation.

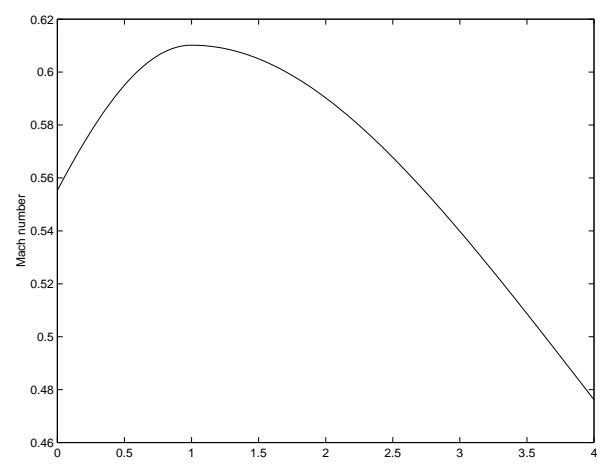

FIG. 3.3. Problem 2: $x \rightarrow M(x)$ for a smooth subsonic flow.

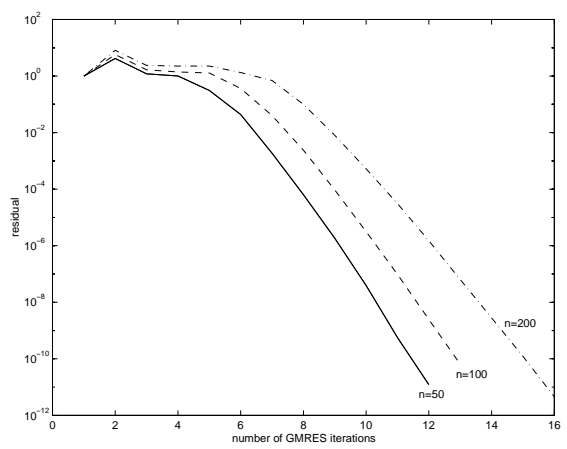

FIG. 3.5. Problem 3: SGS-GMRES(20) method for the transonic flow in Figure 2.2.

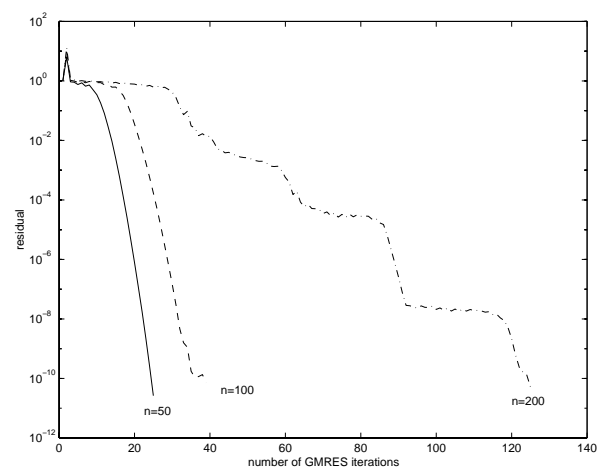

Fig. 3.2. Problem 1: SGS-GMRES(20) method for the subsonic flow in Figure 2.1.

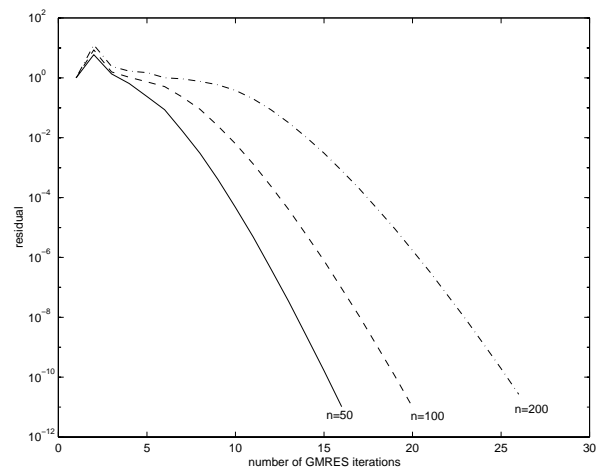

FIG. 3.4. Problem 2: SGS-GMRES(20) method for the subsonic flow in Figure 3.3.

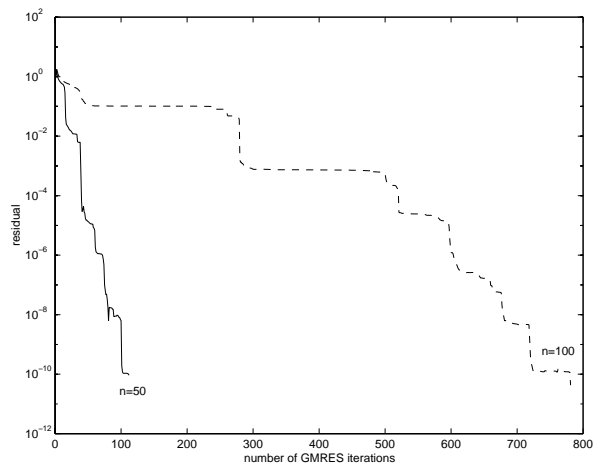

FIG. 3.6. Problem 3: LGS-GMRES(20) method for the transonic flow in Figure 2.2.

1. In the case of the transonic flow in Problem 3 the rate of convergence of the SGS-GMRES(20) method is even higher. We also note that the results presented in Figures 3.4 and 3.5 show a weak dependence of the rate of convergence on the mesh size $h$. Finally note that in Problem 3 the LGS-GMRES(20) method is much slower than the SGS-GMRES(20) method. 
In the next section we present an analysis which yields some theoretical results on the quality of the block-Gauss-Seidel preconditioner. These theoretical results yield a better understanding of the convergence phenomena that are observed in the numerical experiments above.

4. Convergence analysis of the block-Gauss-Seidel method. For the (block) Gauss-Seidel method many convergence results are known in the literature (e.g., see $[1,2,7,8,23])$. These results apply to certain classes of matrices, like, for example, symmetric positive definite matrices or $M$-matrices. We did not find a convergence analysis which yields a satisfactory result when applied to the linearized discrete one dimensional Euler equations. In this section we present an analysis that partly fills this gap.

For the theoretical analysis we consider the homogeneous Euler equations $f(U)_{x}=$ 0 with a constant solution $(\rho(x), u(x), p(x))=(\rho, u, p)=: \bar{V}$ for all $x$. We consider only data with

$$
\rho>0, \quad p>0, \quad M \in(0,1), \quad \gamma:=1.4 .
$$

The corresponding solution vector in conservative variables is denoted by $\bar{U}^{*}$. The Van Leer discretization method as described in section 2 results in a nonlinear system as in (2.3), (2.4), (2.6) with $Q_{S}=0$. The treatment of the boundary conditions (first order accurate) is such that

$$
F_{0}\left(\bar{U}_{0}^{*}, \bar{U}_{1}^{*}\right)=0, \quad F_{n+1}\left(\bar{U}_{n}^{*}, \bar{U}_{n+1}^{*}\right)=0
$$

holds. Hence, the discrete problem has the constant solution $\bar{U}_{h}^{*}\left(x_{i}\right):=\bar{U}^{*}\left(x_{i}\right), i=$ $0, \ldots, n+1$. To avoid technical complications related to the specific treatment of the boundary conditions we consider the nonlinear system in the interior points only; i.e., as unknowns we take $\mathbf{U}=\left(U_{1}, \ldots, U_{n}\right)^{T} \in \mathbb{R}^{3 n}$, and the system of nonlinear equations is given by

$$
\begin{aligned}
F_{1}\left(U_{1}, U_{2}\right) & :=f^{+}\left(U_{1}\right)-f^{-}\left(U_{1}\right)+f^{-}\left(U_{2}\right)=f^{+}\left(\bar{U}_{0}^{*}\right), \\
F_{i}\left(U_{i-1}, U_{i}, U_{i+1}\right) & :=-f^{+}\left(U_{i-1}\right)+f^{+}\left(U_{i}\right)-f^{-}\left(U_{i}\right)+f^{-}\left(U_{i+1}\right)=0, \quad 2 \leq i \leq n-1, \\
F_{n}\left(U_{n-1}, U_{n}\right) & :=-f^{+}\left(U_{n-1}\right)+f^{+}\left(U_{n}\right)-f^{-}\left(U_{n}\right)=-f^{-}\left(\bar{U}_{n+1}^{*}\right) .
\end{aligned}
$$

The vector $\bar{U}_{h}^{*}\left(x_{i}\right)=\bar{U}^{*}\left(x_{i}\right), i=1, \ldots, n$, is a solution of this nonlinear system of equations. The Jacobian system

$$
\mathbf{A v}=\mathbf{b}, \quad \mathbf{A}:=D F\left(\overline{\mathbf{U}}_{h}^{*}\right) \in \mathbb{R}^{3 n \times 3 n}
$$

has a block-tridiagonal matrix

$$
\begin{aligned}
& \mathbf{A}=\operatorname{blocktridiag}\left(-A^{+}, A^{+}-A^{-}, A^{-}\right)_{1 \leq i \leq n}, \\
& A^{+}:=D f^{+}\left(\bar{U}_{h}^{*}\right) \in \mathbb{R}^{3 \times 3}, \quad A^{-}:=D f^{-}\left(\bar{U}_{h}^{*}\right) \in \mathbb{R}^{3 \times 3} .
\end{aligned}
$$

The eigenvalues of $A^{ \pm}$are denoted by $\lambda_{i}^{ \pm}, i=1,2,3$. The Van Leer splitting has been constructed in such a way that both $A^{+}$and $A^{-}$have one zero eigenvalue: $\lambda_{1}^{+}=\lambda_{1}^{-}=0$. The other eigenvalues $\lambda_{2}^{+}, \lambda_{3}^{+}$of $A^{+}$and $\lambda_{2}^{-}, \lambda_{3}^{-}$of $A^{-}$are strictly positive and strictly negative, respectively. For these eigenvalues explicit formulas in terms of $c$ and $M$ are known $[11,28]$. 
Using MAPLE, one obtains

$$
\operatorname{det}\left(A^{+}-A^{-}\right)=\frac{c^{3}}{24}\left(M^{6}-15 M^{4}+3 M^{2}+11\right) .
$$

The polynomial in $M$ on the right-hand side has no zeros for $M \in(-1,1)$. Hence (cf. (4.1)) the matrix $A^{+}-A^{-}$is nonsingular. The matrix

$$
B=B\left(\bar{U}_{h}^{*}\right):=-\left(A^{+}-A^{-}\right)^{-1} A^{-}
$$

plays an important role in the analysis. From $\operatorname{ker}(B)=\operatorname{ker}\left(A^{-}\right)$and $\operatorname{ker}(I-B)=$ $\operatorname{ker}\left(A^{+}\right)$it follows that

$$
\sigma(B)=\{1,0, \mu(\rho, c, M)\}
$$

Using MAPLE, an explicit representation for $B$ can be obtained. The resulting formulas are rather long and not relevant here. We note only that from these formulas it immediately follows that $B$ can be factorized as

$$
B=E \tilde{B}(M) E^{-1}, \quad E=\operatorname{diag}\left(1, c, c^{2}\right),
$$

with a matrix $\tilde{B}(M)$ which depends only on $M$. Hence, the eigenvalue $\mu$ of $B$ in (4.6) depends only on $M$. A further MAPLE computation yields a representation of an eigenvector basis of the matrix $\tilde{B}$ :

$$
\begin{aligned}
\tilde{B} X & =X \operatorname{diag}(1,0, \mu(M)), \\
X & =\left(\begin{array}{ccc}
1 & 1 & 1 \\
\frac{M^{2}+4 M-5}{M+9} & \frac{M^{2}-4 M-5}{M-9} & \frac{6 M}{11} \\
\frac{7 M^{3}-7 M^{2}+5 M+275}{14(M+9)} & \frac{7 M^{3}+7 M^{2}+5 M-275}{14(M-9)} & \frac{16 M^{2}}{77}
\end{array}\right), \\
\mu(M) & =\frac{1}{2} \frac{M^{4}-14 M^{2}+24 M-11}{M^{4}-14 M^{2}-11} .
\end{aligned}
$$

The function $M \rightarrow \mu(M)$ is shown in Figure 4.1. An important observation is that for a large range of Mach numbers $M \in\left[M_{0}, 1\right]$ the eigenvalue $\mu(M)$ is small (e.g., $\mu(M) \in[0,0.1]$ for $M \in[0.5,1])$. The condition number of the matrix $X$ is bounded uniformly in $M \in[0,1]$. The function $M \rightarrow\|X\|_{2}\left\|X^{-1}\right\|_{2}$ is given in Figure 4.2.

In the remainder of this section we analyze block-Gauss-Seidel methods applied to the system (4.3). For any block-tridiagonal matrix $\mathbf{C}=\operatorname{blocktridiag}\left(C_{l}, C_{d}, C_{u}\right)$ we introduce the decomposition $\mathbf{C}=\mathbf{D}-\mathbf{L}-\mathbf{U}$ with $\mathbf{D}:=\operatorname{blockdiag}\left(C_{d}\right)$ and strictly lower and upper triangular matrices $\mathbf{L}$ and $\mathbf{U}$, respectively. We assume that the matrix $\mathbf{D}$ is nonsingular and define the lexicographic and symmetric Gauss-Seidel preconditioners:

$$
\mathbf{W}_{C}^{L G S}:=\mathbf{D}-\mathbf{L}, \quad \mathbf{W}_{C}^{S G S}:=(\mathbf{D}-\mathbf{L}) \mathbf{D}^{-1}(\mathbf{D}-\mathbf{U}) .
$$

Below, the symbol $\mathbf{W}_{C}$ is used to denote both $\mathbf{W}_{C}^{L G S}$ and $\mathbf{W}_{C}^{S G S}$; i.e., statements involving $\mathbf{W}_{C}$ hold both for the lexicographic and the symmetric block-Gauss-Seidel preconditioner. We apply these preconditioners to the matrix $\mathbf{A}$ in (4.4). The blockGauss-Seidel methods are invariant under block-diagonal scaling, and thus the following result holds. 


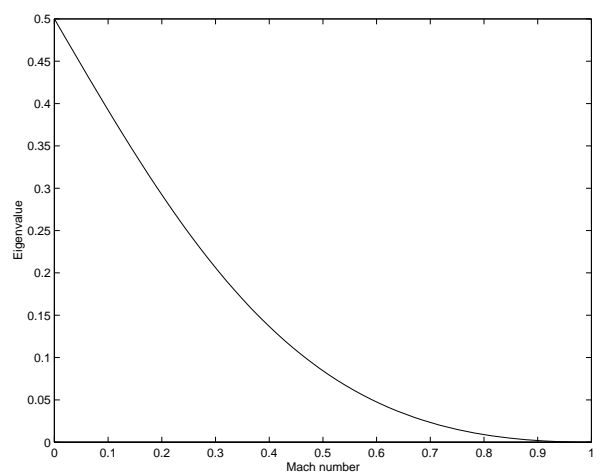

FIG. 4.1. Function $M \rightarrow \mu(M)$.

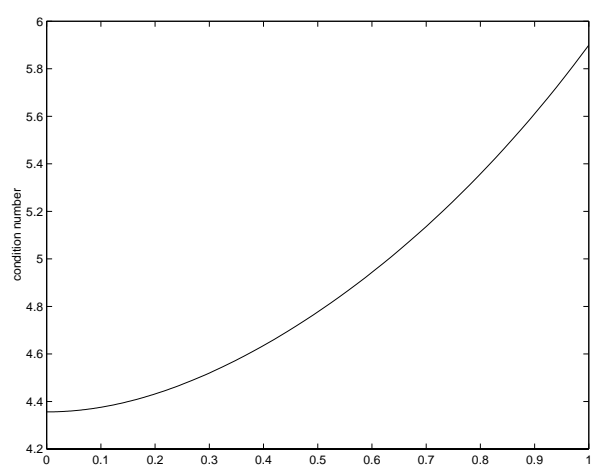

FIG. 4.2. Function $M \rightarrow\|X\|_{2}\left\|X^{-1}\right\|_{2}$.

Lemma 4.1. Define

$$
\tilde{\mathbf{A}}:=\operatorname{blocktridiag}(-(I-B), I,-B)_{1 \leq i \leq n}, \quad \text { with } B \text { as in (4.5). }
$$

Then for the block-Gauss-Seidel preconditioner we have

$$
\mathbf{W}_{A}^{-1} \mathbf{A}=\mathbf{W}_{\tilde{A}}^{-1} \tilde{\mathbf{A}}
$$

We apply a further transformation with the well-conditioned eigenvector basis $X$ of the matrix $\tilde{B}$. For this we introduce

$$
\mathbf{X}:=\operatorname{blockdiag}(X)_{1 \leq i \leq n}, \quad \mathbf{E}:=\operatorname{blockdiag}\left(\begin{array}{ccc}
1 & 0 & 0 \\
0 & c & 0 \\
0 & 0 & c^{2}
\end{array}\right)_{1 \leq i \leq n}, \quad c:=\left(\gamma p \rho^{-1}\right)^{\frac{1}{2}} .
$$

\section{Lemma 4.2. Define}

$$
\hat{\mathbf{A}}:=\operatorname{blocktridiag}\left(-\left(\begin{array}{ccc}
0 & & \emptyset \\
& 1 & \\
\emptyset & & 1-\mu
\end{array}\right),\left(\begin{array}{lll}
1 & & \emptyset \\
& 1 & \\
\emptyset & & 1
\end{array}\right),-\left(\begin{array}{ccc}
1 & & \emptyset \\
& 0 & \\
\emptyset & & \mu
\end{array}\right)\right) \in \mathbb{R}^{3 n \times 3 n}
$$

with $\mu=\mu(M)$ as in (4.9). Then

$$
\mathbf{W}_{A}^{-1} \mathbf{A}=\mathbf{E X} \mathbf{W}_{\hat{A}}^{-1} \hat{\mathbf{A}} \mathbf{X}^{-1} \mathbf{E}^{-1}
$$

holds.

Proof. This follows from

$$
\tilde{\mathbf{A}}=\mathbf{E X} \hat{\mathbf{A}} \mathbf{X}^{-1} \mathbf{E}^{-1}, \quad \mathbf{W}_{\tilde{A}}=\mathbf{E X W} \mathbf{W}_{\hat{A}} \mathbf{X}^{-1} \mathbf{E}^{-1},
$$

and the result in Lemma 4.1.

From Lemma 4.2 it follows that $\sigma\left(\mathbf{W}_{A}^{-1} \mathbf{A}\right)=\sigma\left(\mathbf{W}_{\hat{A}}^{-1} \hat{\mathbf{A}}\right)$. However, it is well known that in a setting with strongly nonnormal matrices the eigenvalues (spectral radius) are in general not a good measure for the rate of convergence of an iterative method (cf. $[8,23])$. Because the blocks in the block-tridiagonal matrix $\hat{\mathbf{A}}$ are diagonal, this matrix represents three decoupled systems of dimension $n$, and a block-GaussSeidel method applied to $\hat{\mathbf{A}}$ is the same as a point Gauss-Seidel method. To make 
this more precise we introduce for $\mathbf{H}=\mathbf{D}-\mathbf{L}-\mathbf{U}$ with $\mathbf{D}=\operatorname{diag}(\mathbf{H}), \mathbf{L}$ and $\mathbf{U}$ strictly lower and strictly upper triangular matrices, respectively, the point GaussSeidel splittings

$$
\mathbf{G}_{H}^{L G S}:=\mathbf{D}-\mathbf{L}, \quad \mathbf{G}_{H}^{S G S}:=(\mathbf{D}-\mathbf{L}) \mathbf{D}^{-1}(\mathbf{D}-\mathbf{U}) .
$$

The symbol $\mathbf{G}_{H}$ is used to denote both $\mathbf{G}_{H}^{L G S}$ and $\mathbf{G}_{H}^{S G S}$. Let $\mathbf{P} \in \mathbb{R}^{3 n \times 3 n}$ be the permutation matrix given by

$$
(\mathbf{P x})_{k+3(i-1)}=x_{(k-1) n+i}, \quad k=1,2,3, \quad i=1, \ldots, n .
$$

We introduce the tridiagonal $n \times n$-matrices

$$
\mathbf{L}:=\left(\begin{array}{cccc}
1 & & & \\
-1 & 1 & & \\
& \ddots & \ddots & \\
& & -1 & 1
\end{array}\right), \quad \mathbf{T}=\mathbf{T}_{\mu}:=\left(\begin{array}{cccc}
1 & -\mu & & \\
-(1-\mu) & 1 & \ddots & \\
& \ddots & \ddots & -\mu \\
& & -(1-\mu) & 1
\end{array}\right) .
$$

From the result in Lemma 4.2 one obtains the following.

LEMMA 4.3. The following holds:

$$
\begin{aligned}
& \mathbf{E}^{-1} \mathbf{W}_{A}^{-1} \mathbf{A E}=\mathbf{X P Q P} \mathbf{P}^{-1} \mathbf{X}^{-1} \\
& \text { with } \mathbf{Q}=\left(\begin{array}{ccc}
\mathbf{Q}_{1} & & \emptyset \\
\emptyset & \mathbf{Q}_{2} & \\
& & \mathbf{Q}_{3}
\end{array}\right):=\left(\begin{array}{ccc}
\mathbf{G}_{L^{T}}^{-1} \mathbf{L}^{T} & & \emptyset \\
\emptyset & \mathbf{G}_{L}^{-1} \mathbf{L} & \\
\emptyset & & \mathbf{G}_{T}^{-1} \mathbf{T}
\end{array}\right) \text {. }
\end{aligned}
$$

We now consider a Krylov subspace method applied to the matrix $\mathbf{W}_{A}^{-1} \mathbf{A}$. Let $\mathcal{P}_{k}$ be the space of polynomials of degree less than or equal to $k$ and $\mathcal{P}_{k}^{*}:=\{p \in$ $\left.\mathcal{P}_{k} \mid p(0)=1\right\}$. A Krylov subspace method can be described by a corresponding polynomial $p_{k} \in \mathcal{P}_{k}^{*}$. Based on the result in Lemma 4.3 we use the problem dependent scaled Euclidean norm

$$
\|\mathbf{y}\|_{E}:=\left\|\mathbf{E}^{-1} \mathbf{y}\right\|_{2}, \quad \mathbf{y} \in \mathbb{R}^{3 n}
$$

Let $\kappa_{2}(\mathbf{C}):=\left\|\mathbf{C}^{-1}\right\|_{2}\|\mathbf{C}\|_{2}$ be the spectral condition number. From Lemma 4.3 it follows that

$$
\kappa_{2}(X)^{-1}\left\|p_{k}(\mathbf{Q})\right\|_{2} \leq\left\|p_{k}\left(\mathbf{W}_{A}^{-1} \mathbf{A}\right)\right\|_{E} \leq \kappa_{2}(X)\left\|p_{k}(\mathbf{Q})\right\|_{2}
$$

Since $\kappa_{2}(X)$ is independent of $n$ and uniformly (w.r.t. $M$ ) bounded, the quantity $\left\|p_{k}(\mathbf{Q})\right\|_{2}$ is a reasonable measure for the rate of convergence of the Krylov subspace method applied to $\mathbf{W}_{A}^{-1} \mathbf{A}$. We therefore consider

$$
\left\|p_{k}(\mathbf{Q})\right\|_{2}=\max _{1 \leq i \leq 3}\left\|p_{k}\left(\mathbf{Q}_{i}\right)\right\|_{2} .
$$

In order to derive bounds for $\left\|p_{k}(\mathbf{C})\right\|_{2}, \mathbf{C} \in \mathbb{R}^{n}$, one usually makes the natural assumption that the symmetric part of the matrix $\mathbf{C}$ is positive definite. This assumption is satisfied in our case.

LEMma 4.4. The following holds:

$$
\frac{1}{2} \lambda_{\min }\left(\mathbf{Q}_{i}+\mathbf{Q}_{i}^{T}\right):=\min \left\{\mathbf{y}^{T} \mathbf{Q}_{i} \mathbf{y} \mid \mathbf{y} \in \mathbb{R}^{n},\|\mathbf{y}\|_{2}=1\right\}>0 \quad \text { for } i=1,2,3 .
$$


Proof. Note that for the LGS and the SGS methods we have

$$
\left\|\mathbf{I}-\mathbf{G}_{T}^{-1} \mathbf{T}\right\|_{\infty}<1, \quad\left\|\mathbf{I}-\mathbf{G}_{T}^{-1} \mathbf{T}\right\|_{1}<1 .
$$

From this it follows that

$$
\rho\left(\mathbf{I}-\frac{1}{2}\left(\mathbf{G}_{T}^{-1} \mathbf{T}+\left(\mathbf{G}_{T}^{-1} \mathbf{T}\right)^{T}\right)\right) \leq \frac{1}{2}\left\|\mathbf{I}-\mathbf{G}_{T}^{-1} \mathbf{T}\right\|_{\infty}+\frac{1}{2}\left\|\mathbf{I}-\mathbf{G}_{T}^{-1} \mathbf{T}\right\|_{1}<1,
$$

and thus

$$
\lambda_{\min }\left(\frac{1}{2}\left(\mathbf{Q}_{3}+\mathbf{Q}_{3}^{T}\right)\right)>0
$$

Similar arguments can be used to prove the results for $i=1$ and $i=2$.

In the literature one can find analyses in which for several classes of Krylov subspace methods, under the assumption that the symmetric part of $\mathbf{C}$ is positive definite, bounds for $\left\|p_{k}(\mathbf{C})\right\|_{2}$ in terms of the quantity

$$
\xi(\mathbf{C}):=\frac{\|\mathbf{C}\|_{2}}{\frac{1}{2} \lambda_{\min }\left(\mathbf{C}+\mathbf{C}^{T}\right)}
$$

are derived (cf. $[9,10,22])$. These bounds are in general very pessimistic but indicate that if $\xi(\mathbf{C})$ is "small" (i.e., close to one), one can expect fast convergence of the Krylov subspace method applied to C. Another interesting quantity related to the rate of convergence is the spectral condition number $\kappa_{2}(\mathbf{C})$. Note that

$$
1 \leq \kappa_{2}(\mathbf{C}) \leq \xi(\mathbf{C})
$$

holds. Based on this and on the result in (4.11) we take

$$
\xi_{\max }:=\max _{1 \leq i \leq 3} \xi\left(\mathbf{Q}_{i}\right), \quad \kappa_{\max }:=\max _{1 \leq i \leq 3} \kappa_{2}\left(\mathbf{Q}_{i}\right)
$$

as measures for the quality of the block-Gauss-Seidel preconditioner.

We now distinguish between the LGS and SGS methods.

TheOREm 4.5. For the lexicographic block-Gauss-Seidel method we have

$$
\mathbf{G}=\mathbf{G}^{L G S}, \quad \xi_{\max }=\max \left\{\xi\left(\mathbf{Q}_{1}\right), \xi\left(\mathbf{Q}_{3}\right)\right\}, \quad \kappa_{\max }=\max \left\{\kappa_{2}\left(\mathbf{Q}_{1}\right), \kappa_{2}\left(\mathbf{Q}_{3}\right)\right\} .
$$

For the symmetric block-Gauss-Seidel method we have

$$
\mathbf{G}=\mathbf{G}^{S G S}, \quad \xi_{\max }=\xi\left(\mathbf{Q}_{3}\right), \quad \kappa_{\max }=\kappa_{2}\left(\mathbf{Q}_{3}\right) .
$$

Proof. For the LGS method we have

$$
\mathbf{G}_{L^{T}}=\mathbf{I}, \quad \mathbf{G}_{L}=\mathbf{L}
$$

and for the SGS method

$$
\mathbf{G}_{L^{T}}=\mathbf{L}^{T}, \quad \mathbf{G}_{L}=\mathbf{L} .
$$

Hence $\mathbf{Q}_{2}=\mathbf{I}$ for the LGS and for the SGS methods, and $\mathbf{Q}_{1}=\mathbf{I}$ for the SGS method. 


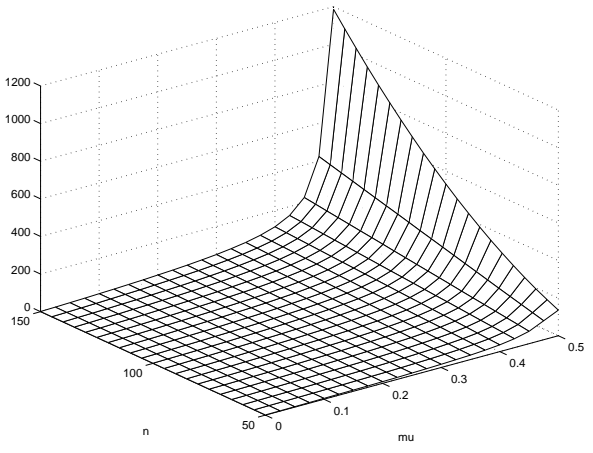

FIG. 4.3. $\kappa_{2}\left(\mathbf{G}_{T}^{-1} \mathbf{T}\right)$ for $\mathbf{G}_{T}=\mathbf{G}_{T}^{S G S}$.

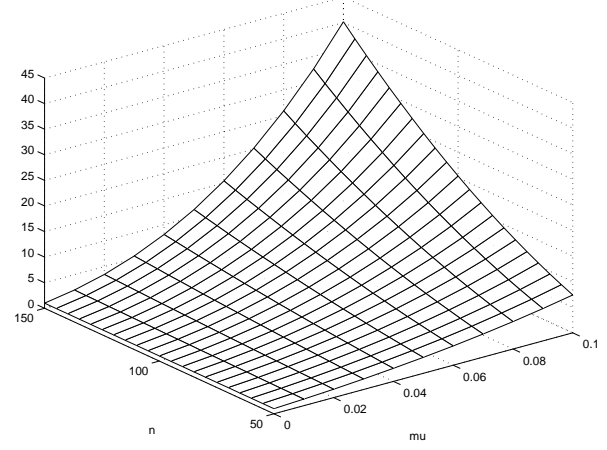

Fig. 4.5. $\xi\left(\mathbf{G}_{T}^{-1} \mathbf{T}\right)$ for $\mathbf{G}_{T}=\mathbf{G}_{T}^{S G S}$.

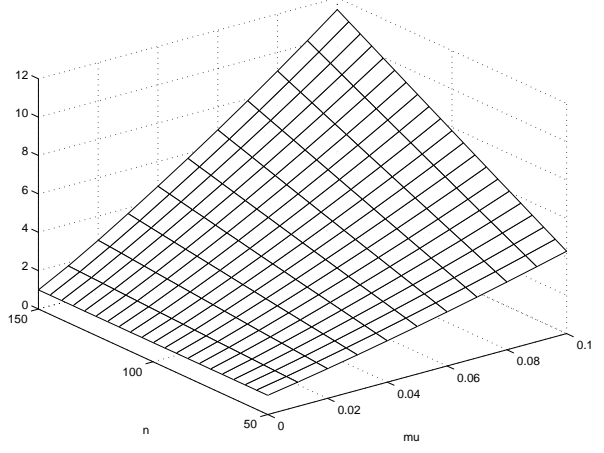

FIG. 4.4. $\kappa_{2}\left(\mathbf{G}_{T}^{-1} \mathbf{T}\right)$ for $\mathbf{G}_{T}=\mathbf{G}_{T}^{S G S}$.

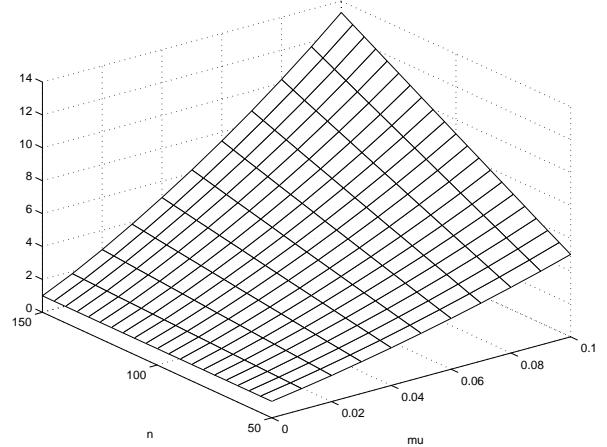

FIG. 4.6. $\kappa_{2}\left(\mathbf{G}_{T}^{-1} \mathbf{T}\right)$ for $\mathbf{G}_{T}=\mathbf{G}_{T}^{L G S}$.

In Figures 4.3 and 4.4 for the SGS method the dependence of $\kappa_{2}\left(\mathbf{Q}_{3}\right)=\kappa_{2}\left(\mathbf{G}_{T}^{-1} \mathbf{T}\right)$ on $\mu$ and $n$ is shown.

From these figures and the result in (4.14) it follows that for $\mu \in\left(0, \mu_{0}\right)$ with $\mu_{0} \ll \frac{1}{2}$ the function $n \rightarrow \kappa_{\max }(n)$ increases only slowly. Hence, for "small" $\mu$-values the SGS-preconditioned matrix has a corresponding $\kappa_{\max }$-value which is small, even for "large" $n$-values. Now note that the dependence of $\mu$ on the Mach number $M$ is as in (4.9) (Figure 4.1), and thus for a large range of Mach numbers $M \in\left[M_{0}, 1\right]$ the corresponding $\mu(M)$-values are (very) small, and thus the condition number $\kappa_{\max }$ is small, too. In Figure 4.5 for the SGS method we show, for small $\mu$-values, the dependence of $\xi_{\max }=\xi\left(\mathbf{G}_{T}^{-1} \mathbf{T}\right)$ on $\mu$ and $n$. Note that for small $\mu$-values the function $n \rightarrow \xi_{\max }(n)$ increases slowly, too. These observations yield some theoretical explanation of the fast convergence of the SGS-GMRES(20) method in Problems 1 and 2 as compared to the diffusion problem (cf. Figures 3.1, 3.2, 3.4), and of the fact that in Problem 2 (Figure 3.4) the rate of convergence is much higher than in Problem 1 (Figure 3.2).

For the LGS method the term $\xi\left(\mathbf{Q}_{1}\right)$ in (4.13) has to be taken into account. For this term we have

$$
\xi\left(\mathbf{Q}_{1}\right)=\frac{\left\|\mathbf{L}^{T}\right\|_{2}}{\frac{1}{2} \lambda_{\min }\left(\mathbf{L}+\mathbf{L}^{T}\right)} \approx 4\left(\frac{n}{\pi}\right)^{2}
$$

which is, independently of $\mu$, large if $n$ is large. This gives a theoretical justification of 
the intuitive conjecture that for a subsonic or transonic one dimensional flow problem with characteristics going in both directions the SGS method should perform (much) better than the LGS method (cf. also the large difference in the rates of convergence in Figures 3.5, 3.6).

The result in (4.14) relates the quality measure $\kappa_{\max }$ of the SGS-method to the condition number $\kappa_{2}\left(\mathbf{G}_{T}^{-1} \mathbf{T}\right)$. The behavior of the function $(\mu, n) \rightarrow \kappa_{2}\left(\mathbf{G}_{T}^{-1} \mathbf{T}\right)$ is shown in Figures 4.3 and 4.4. An important observation is that for "small" $\mu$ values these condition numbers are small. The same holds for the LGS method (cf. Figure 4.6). One can derive (fairly sharp) bounds for $\kappa_{2}\left(\mathbf{G}_{T}^{-1} \mathbf{T}\right)$, which show the dependence of this condition number on $n$ and $\mu$. Here we present such a result for the simplest case, namely for the LGS method. For completeness a proof is given in the appendix. A similar result can be shown to hold for the SGS method.

THEOREM 4.6. Let $\mathbf{G}=\mathbf{G}_{T}^{L G S}$ be the $L G S$ preconditioner for the matrix $\mathbf{T}=$ $\mathbf{T}_{\mu} \in \mathbb{R}^{n \times n}$. For the condition number of the preconditioned matrix the following holds for $\mu \in\left[0, \frac{1}{2}\right]$ :

$$
\begin{aligned}
\left\|\mathbf{G}^{-1} \mathbf{T}\right\|_{2}\left\|\mathbf{T}^{-1} \mathbf{G}\right\|_{2} & \leq\left(1+\min \left\{\frac{\mu}{h}, 1\right\}\right) \frac{2 \delta_{\mu}}{1-2 \mu}\left(\frac{\mu}{h}+1+\frac{\mu \delta_{\mu}}{1-2 \mu \delta_{\mu}} \frac{1}{\sqrt{h}}\right), \\
\text { with } h & =\frac{1}{n+1}, \quad \delta_{\mu}=\min \left\{1, \frac{1-2 \mu}{8 \mu} \frac{1}{h}\right\} .
\end{aligned}
$$

Remark 1. In our model problem we are interested in the case $\mu \ll \frac{1}{2}$ (e.g., $\mu \in(0,0.1))$ and $h \ll 1$. For this case we have $\delta_{\mu}=1$, and we obtain the following bound for the condition number:

$$
\left\|\mathbf{G}^{-1} \mathbf{T}\right\|_{2}\left\|\mathbf{T}^{-1} \mathbf{G}\right\|_{2} \lesssim\left\{\begin{array}{lll}
2\left(1+\frac{\mu}{h}\right)^{2} & \text { if } & \frac{\mu}{h}<1 \\
4\left(1+\frac{\mu}{h}\right) & \text { if } & \frac{\mu}{h} \geq 1 .
\end{array}\right.
$$

This bound clearly shows that for small $\mu$ there is (at worst) only a slow growth in the condition number as a function of $n=h^{-1}-1$.

Remark 2 . We briefly comment on the very high rate of convergence of the SGSGMRES(20) method for the transonic flow problem in section 3 (Figures 2.2 and 3.5). In part of the domain the flow is supersonic $(M>1)$, and in another part of the domain the flow is subsonic with Mach numbers $M \in(0.6,1)$. The upwind discretization in the supersonic part of the domain results in a block lower triangular matrix. Hence in this part of the domain the information is propagated exactly by the symmetric block-Gauss-Seidel method. In the subsonic part of the domain the Mach numbers are $\geq 0.6$, and thus the corresponding $\mu(M)$-values lie in the interval $[0,0.05]$. The analysis in this section shows that in such a case if we freeze the coefficients, the SGS method can be expected to be a very effective preconditioner. At the "critical" points $x=1$ and $x=3$ we do not have a smooth behavior, and this results in a low dimensional subspace in which the Gauss-Seidel preconditioner may perform relatively poorly. Due to its very low dimension the error components in this subspace can be reduced effectively by the GMRES method. These arguments give some heuristic explanation of the convergence behavior shown in Figure 3.5. A rigorous analysis for the transonic case is still lacking.

Remark 3. We briefly comment on possible topics for further research towards two dimensional problems. Consider a stationary two dimensional Euler equation that is discretized on a uniform square grid using the Van Leer flux vector-splitting method. The resulting nonlinear problem is linearized at the discrete solution. For 
the analysis we assume that $V=(\rho, u, v, p)$ is constant as a function of the space variable. In stencil notation the discrete problem has the structure (cf. (4.4))

$$
\left[\begin{array}{ccc} 
& B^{-} \\
-A^{+} & A^{+}-A^{-}+B^{+}-B^{-} & A^{-} \\
& -B^{+}
\end{array}\right]
$$

with $A^{ \pm}, B^{ \pm} \in \mathbb{R}^{4 \times 4}$. For the Mach numbers in one direction we use the notation $M_{u}:=\frac{u}{c}, M_{v}:=\frac{v}{c}$. We consider only $M_{u} \geq 0, M_{v} \geq 0$.

In the supersonic case $M_{u} \geq 1, M_{v} \geq 1$ we have $B^{-}=A^{-}=0$, and thus the matrix is block lower triangular. Hence the block-Gauss-Seidel method is a direct solver.

For the supersonic case $M_{u} \in(0,1), M_{v} \geq 1$ we have that $B^{-}=0$, and thus the $x$ line block-Gauss-Seidel method is a direct solver. To analyze convergence properties of the symmetric block-Gauss-Seidel method (which is not a direct solver) one has to investigate the SGS method applied to the matrix

$$
\text { blocktridiag }\left(-A^{+}, A^{+}-A^{-}+B^{+}, A^{-}\right) \text {. }
$$

This corresponds to a one dimensional problem, and thus for the analysis one can try to use the same approach used in section 4. Note, however, that the matrix $A^{+} \in \mathbb{R}^{4 \times 4}$ differs from the one in (4.4).

For the subsonic case $M_{u}=M_{v} \in(0,1)$ one has nice symmetry properties. We have $P B^{ \pm}=A^{ \pm}$with a simple permutation matrix $P$. Hence properties of the matrix corresponding to (4.15) essentially depend only on those of $A^{+}$and $A^{-}$. Suitable transformations (as in section 4) based on the known eigenvector bases of $A^{ \pm}$may help to determine some of these properties.

Appendix. Proof of Theorem 4.6. In this appendix we give a proof of the result in Theorem 4.6. We consider the tridiagonal matrix $\mathbf{T}=\mathbf{T}_{\mu}$ as (4.10) with $\mu \in\left(0, \frac{1}{2}\right)$, and for the preconditioner we take the LGS method:

$$
\mathbf{G}=\operatorname{tridiag}(-(1-\mu), 1,0) \in \mathbb{R}^{n \times n} .
$$

In Figure 4.6 we showed the numerically computed values of the function $(\mu, n) \rightarrow$ $\kappa_{2}\left(\mathbf{G}^{-1} \mathbf{T}\right)$. In this section we derive a rigorous (sharp) bound for this condition number, which shows its dependence on $\mu$ and $h=1 /(n+1)$.

We use the notation

$$
\mathbf{S}=\left(\begin{array}{cccc}
0 & 1 & & \\
& 0 & \ddots & \\
& & \ddots & 1 \\
& & & 0
\end{array}\right) \in \mathbb{R}^{n \times n}, \quad \mathbf{W}=\mathbf{I}-\mathbf{S}^{T} .
$$

Lemma A.1. The following holds:

$$
\left\|\mathbf{G}^{-1} \mathbf{T}\right\|_{2} \leq 1+\min \left\{\frac{\mu}{h}, 1\right\} .
$$

Proof. Using $\mathbf{T}=\mathbf{G}-\mu \mathbf{S}$, we obtain

$$
\begin{aligned}
\left\|\mathbf{G}^{-1} \mathbf{T}\right\|_{2} & =\left\|\mathbf{I}-\mu \mathbf{G}^{-1} \mathbf{S}\right\|_{2} \leq 1+\mu\left(\left\|\mathbf{G}^{-1} \mathbf{S}\right\|_{\infty}\left\|\mathbf{G}^{-1} \mathbf{S}\right\|_{1}\right)^{\frac{1}{2}} \\
& \leq 1+\mu \sum_{k=0}^{n-1}(1-\mu)^{k} \leq 1+\min \{\mu n, 1\}
\end{aligned}
$$


and thus the result of this lemma holds.

We now derive a bound for $\left\|\mathbf{T}^{-1} \mathbf{G}\right\|_{2}$. First we note that $\mathbf{T}=\mathbf{G}-\mu \mathbf{S}$ is a weakly regular splitting; i.e., $\mathbf{G}^{-1} \geq 0$ and $\mu \mathbf{G}^{-1} \mathbf{S} \geq 0$ hold. Moreover, $\mathbf{T}^{-1} \geq 0$ holds, and thus $\mu \rho\left(\mathbf{G}^{-1} \mathbf{S}\right)<1$. From this we obtain that $\mathbf{T}^{-1} \mathbf{G}$ is a positive matrix:

$$
\mathbf{T}^{-1} \mathbf{G}=\left(\mathbf{I}-\mu \mathbf{G}^{-1} \mathbf{S}\right)^{-1}=\sum_{k=0}^{\infty}\left(\mu \mathbf{G}^{-1} \mathbf{S}\right)^{k} \geq 0 .
$$

In our analysis we use the numerical radius

$$
r(\mathbf{A}):=\max \left\{\left|\mathbf{x}^{H} \mathbf{A} \mathbf{x}\right| \mid \mathbf{x} \in \mathbb{C}^{n},\|\mathbf{x}\|_{2}=1\right\} .
$$

We also use the following properties:

$$
\begin{aligned}
\|\mathbf{A}\|_{2} & \leq 2 r(\mathbf{A}), \\
r(\mathbf{A}) & =\frac{1}{2} \rho\left(\mathbf{A}+\mathbf{A}^{T}\right) \quad \text { if } \quad \mathbf{A} \geq 0 .
\end{aligned}
$$

Using $\mathbf{G}=\mathbf{I}-(1-\mu) \mathbf{S}^{T}=\mathbf{W}+\mu \mathbf{S}^{T}$, we get

$$
\begin{aligned}
\left\|\mathbf{T}^{-1} \mathbf{G}\right\|_{2} & \leq 2 r\left(\mathbf{T}^{-1} \mathbf{G}\right)=\rho\left(\mathbf{T}^{-1} \mathbf{G}+\mathbf{G}^{T} \mathbf{T}^{-T}\right) \\
& =\rho\left(\left(\mathbf{T}^{-1} \mathbf{W}+\mathbf{W}^{T} \mathbf{T}^{-T}\right)+\mu\left(\mathbf{T}^{-1} \mathbf{S}^{T}+\mathbf{S} \mathbf{T}^{-T}\right)\right) \\
& \leq \rho\left(\mathbf{T}^{-1} \mathbf{W}+\mathbf{W}^{T} \mathbf{T}^{-T}\right)+\mu \rho\left(\mathbf{T}^{-1} \mathbf{S}^{T}+\mathbf{S} \mathbf{T}^{-T}\right) .
\end{aligned}
$$

In the following two lemmas we derive bounds for the two terms in (A.1).

Lemma A.2. The following holds:

$$
\begin{aligned}
\mu \rho\left(\mathbf{T}^{-1} \mathbf{S}^{T}+\mathbf{S T}^{-T}\right) & \leq \frac{2 \delta_{\mu}}{1-2 \mu} \frac{\mu}{h} \\
\text { with } \quad \delta_{\mu} & :=\min \left\{1, \frac{1-2 \mu}{8 \mu} \frac{1}{h}\right\} .
\end{aligned}
$$

Proof. Note that

$$
\rho\left(\mathbf{T}^{-1} \mathbf{S}^{T}+\mathbf{S} \mathbf{T}^{-T}\right) \leq\left\|\mathbf{T}^{-1} \mathbf{S}^{T}+\mathbf{S} \mathbf{T}^{-T}\right\|_{\infty} \leq\left\|\mathbf{T}^{-1}\right\|_{\infty}+\left\|\mathbf{T}^{-T}\right\|_{\infty} .
$$

We derive a bound on $\left\|\mathbf{T}^{-1}\right\|_{\infty}$ using $\mathbf{T}^{-1} \geq 0$ and an appropriate barrier function. The difference operator corresponding to $\mathbf{T}$ is given by

$$
\begin{aligned}
& {[T]_{x_{i}}=\mu\left[\begin{array}{lll}
-1 & 2 & -1
\end{array}\right]_{x_{i}}+(1-2 \mu)\left[\begin{array}{lll}
-1 & 1 & 0
\end{array}\right]_{x_{i}}} \\
& =(1-2 \mu) h\left(\frac{\varepsilon}{h^{2}}\left[\begin{array}{lll}
-1 & 2 & -1
\end{array}\right]_{x_{i}}+\frac{1}{h}\left[\begin{array}{lll}
-1 & 1 & 0
\end{array}\right]_{x_{i}}\right),
\end{aligned}
$$

with $x_{i}=i h, 0 \leq i \leq n+1$, and $\varepsilon=\frac{\mu h}{1-2 \mu} \in(0, \infty)$. To obtain a suitable barrier function we consider the boundary value problem

$$
-\varepsilon u^{\prime \prime}(x)+u^{\prime}(x)=1, \quad x \in(0,1), \quad u(0)=u(1)=0,
$$

with solution given by

$$
\bar{u}(x)=x-\frac{\exp \left(\frac{x}{\varepsilon}\right)-1}{\exp \left(\frac{1}{\varepsilon}\right)-1} \in[0,1] .
$$


For $x \in(0,1)$ and $m \geq 2, \bar{u}^{(m)}(x) \leq 0$ holds. Using this, it follows from a Taylor expansion that

$$
[T]_{x_{i}} \bar{u} \geq(1-2 \mu) h\left(-\varepsilon \bar{u}^{\prime \prime}\left(x_{i}\right)+\bar{u}^{\prime}\left(x_{i}\right)\right)=(1-2 \mu) h .
$$

From this and the fact that $\mathbf{T}$ is inverse positive we obtain

$$
\left\|\mathbf{T}^{-1}\right\|_{\infty} \leq \frac{\|\bar{u}\|_{\infty,[0,1]}}{(1-2 \mu) h}
$$

We introduce the notation $z_{\varepsilon}:=\varepsilon\left(\exp \left(\frac{1}{\varepsilon}\right)-1\right)$. A simple computation yields that on $[0,1]$ the function $\bar{u}$ attains its maximum at $x=\varepsilon \ln z_{\varepsilon}$, and this maximum is given by

$$
\|\bar{u}\|_{\infty,[0,1]}=\varepsilon\left(\ln z_{\varepsilon}+z_{\varepsilon}^{-1}-1\right)=: m(\varepsilon) .
$$

On $(0, \infty)$ the function $\varepsilon \rightarrow m(\varepsilon)$ has the following properties:

$$
\begin{gathered}
\lim _{\varepsilon \downarrow 0} m(\varepsilon)=1, \quad m^{\prime}(\varepsilon)<0, \quad \lim _{\varepsilon \rightarrow \infty} m(\varepsilon)=0, \\
\lim _{\varepsilon \downarrow 0} \varepsilon m(\varepsilon)=0, \quad(\varepsilon m(\varepsilon))^{\prime}>0, \quad \lim _{\varepsilon \rightarrow \infty} \varepsilon m(\varepsilon)=\frac{1}{8} .
\end{gathered}
$$

It follows that

$$
\begin{aligned}
\left\|\mathbf{T}^{-1}\right\|_{\infty} & \leq \frac{1}{(1-2 \mu) h} m(\varepsilon) \leq \frac{1}{(1-2 \mu) h}, \\
\left\|\mathbf{T}^{-1}\right\|_{\infty} & \leq \frac{\varepsilon^{-1}}{(1-2 \mu) h} \varepsilon m(\varepsilon) \leq \frac{1}{\mu h^{2}} \frac{1}{8}
\end{aligned}
$$

and thus

$$
\left\|\mathbf{T}^{-1}\right\|_{\infty} \leq \frac{1}{(1-2 \mu) h} \delta_{\mu}
$$

The same bound can be derived for $\left\|\mathbf{T}^{-T}\right\|_{\infty}$ if one uses the (adjoint) equation $-\varepsilon u^{\prime \prime}-$ $u^{\prime}=1$. These bounds in combination with (A.2) prove the result.

Lemma A.3. The following holds, with $\delta_{\mu}$ as in Lemma A.2:

$$
\rho\left(\mathbf{T}^{-1} \mathbf{W}+\mathbf{W}^{T} \mathbf{T}^{-T}\right) \leq \frac{2 \delta_{\mu}}{1-2 \mu}\left(1+\frac{\mu \delta_{\mu}}{1-2 \mu+\mu \delta_{\mu}} h^{-\frac{1}{2}}\right)
$$

Proof. We use the notation

$$
\begin{aligned}
& \xi=\frac{\mu}{1-\mu}, \quad \mathbf{1}=(1, \ldots, 1)^{T} \in \mathbb{R}^{n}, \quad \mathbf{e}_{1}=(1,0, \ldots, 0)^{T} \in \mathbb{R}^{n}, \\
& \mathbf{x}=(\mathbf{I}-\xi \mathbf{S})^{-1} \mathbf{1}, \quad \mathbf{y}=\left(\mathbf{I}-\xi \mathbf{S}^{T}\right)^{-1} \mathbf{e}_{1}=\left(1, \xi, \xi^{2}, \ldots, \xi^{n-1}\right)^{T}, \\
& \beta=\|\mathbf{y}\|_{1}=\sum_{k=0}^{n-1} \xi^{k}, \quad \tau=\frac{\xi}{1+\xi \beta} .
\end{aligned}
$$


Note that

$$
\begin{aligned}
\mathbf{T}^{-1} \mathbf{W} & =\left(\mathbf{W}-\mu\left(\mathbf{S}-\mathbf{S}^{T}\right)\right)^{-1} \mathbf{W}=\left(\mathbf{I}-\mu \mathbf{W}^{-1}\left(\mathbf{S}-\mathbf{S}^{T}\right)\right)^{-1} \\
& =\left(\mathbf{I}-\mu\left(\mathbf{I}+\mathbf{S}-\mathbf{1 e}_{1}^{T}\right)\right)^{-1}=\frac{1}{1-\mu}\left(\mathbf{I}-\xi \mathbf{S}+\xi \mathbf{1} \mathbf{e}_{1}^{T}\right)^{-1} \\
& =\frac{1}{1-\mu}\left(\mathbf{I}+\xi \mathbf{x} \mathbf{e}_{1}^{T}\right)^{-1}(\mathbf{I}-\xi \mathbf{S})^{-1}=\frac{1}{1-\mu}\left(\mathbf{I}-\tau \mathbf{x} \mathbf{e}_{1}^{T}\right)(\mathbf{I}-\xi \mathbf{S})^{-1} \\
& =\frac{1}{1-\mu}\left((\mathbf{I}-\xi \mathbf{S})^{-1}-\tau \mathbf{x} \mathbf{y}^{T}\right) .
\end{aligned}
$$

Using

$$
\begin{aligned}
\left\|(\mathbf{I}-\xi \mathbf{S})^{-1}\right\|_{2} & \leq\left(\left\|(\mathbf{I}-\xi \mathbf{S})^{-1}\right\|_{\infty}\left\|(\mathbf{I}-\xi \mathbf{S})^{-1}\right\|_{1}\right)^{\frac{1}{2}}=\beta, \\
\|\mathbf{x}\|_{2} & \leq\left\|(\mathbf{I}-\xi \mathbf{S})^{-1}\right\|_{2}\|\mathbf{1}\|_{2} \leq \beta \sqrt{n} \leq \beta h^{-\frac{1}{2}}, \\
\|\mathbf{y}\|_{2} & \leq\left\|(\mathbf{I}-\xi \mathbf{S})^{-1}\right\|_{2}\left\|\mathbf{e}_{1}\right\|_{2} \leq \beta,
\end{aligned}
$$

we obtain

$$
\begin{aligned}
\rho\left(\mathbf{T}^{-1} \mathbf{W}+\mathbf{W}^{T} \mathbf{T}^{-T}\right) & =\frac{1}{1-\mu} \rho\left((\mathbf{I}-\xi \mathbf{S})^{-1}+\left(\mathbf{I}-\xi \mathbf{S}^{T}\right)^{-1}-\tau\left(\mathbf{x} \mathbf{y}^{T}+\mathbf{y} \mathbf{x}^{T}\right)\right) \\
& \leq \frac{2}{1-\mu}\left(\left\|(\mathbf{I}-\xi \mathbf{S})^{-1}\right\|_{2}+\tau\|\mathbf{x}\|_{2}\|\mathbf{y}\|_{2}\right) \\
& \leq \frac{2 \beta}{1-\mu}\left(1+\frac{\xi \beta}{1+\xi \beta} h^{-\frac{1}{2}}\right)
\end{aligned}
$$

We use

$$
\begin{aligned}
\beta & \leq \min \left\{\frac{1}{1-\xi}, n\right\} \leq \frac{1-\mu}{1-2 \mu} \min \left\{1, \frac{1-2 \mu}{1-\mu} h^{-1}\right\} \\
& \leq \frac{1-\mu}{1-2 \mu} \min \left\{1, \frac{1-2 \mu}{8 \mu} h^{-1}\right\}=\frac{1-\mu}{1-2 \mu} \delta_{\mu} .
\end{aligned}
$$

Hence

$$
\frac{2 \beta}{1-\mu} \leq \frac{2 \delta_{\mu}}{1-2 \mu}
$$

holds. Finally, note that

$$
\frac{\xi \beta}{1+\xi \beta} \leq \frac{\frac{\mu}{1-2 \mu} \delta_{\mu}}{1+\frac{\mu}{1-2 \mu} \delta_{\mu}}=\frac{\mu \delta_{\mu}}{1-2 \mu+\mu \delta_{\mu}} .
$$

Combination of (A.3), (A.4), and (A.5) yields the result.

Substitution of the results of Lemmas A.2 and A.3 into (A.1) yields

$$
\left\|\mathbf{T}^{-1} \mathbf{G}\right\|_{2} \leq \frac{2 \delta_{\mu}}{1-2 \mu}\left(\frac{\mu}{h}+1+\frac{\mu \delta_{\mu}}{1-2 \mu+\mu \delta_{\mu}} \frac{1}{\sqrt{h}}\right) .
$$

Combination of this result with the result of Lemma A.1 shows that the inequality in Theorem 4.6 holds. 


\section{REFERENCES}

[1] O. Axelsson, Iterative Solution Methods, Cambridge University Press, New York, 1994.

[2] J. BEy AND A. REusken, On the convergence of basic iterative methods for convection-diffusion equations, Numer. Linear Algebra Appl., 6 (1999), pp. 329-352.

[3] Ph. BIRKen, Preconditioning GMRES for Steady Compressible Inviscid Flows, Institute für Geometric und Praktische Mathematik Report 212, Rheinisch-Westfälische Technische Hochschule-Aachen, Aachen, Germany, 2002.

[4] F. Bramkamp, J. Ballmann, and S. Müller, Development of a flow solver employing local adaptation based on multiscale analysis on B-spline grids, in Proceedings of the 8th Annual Conference of the CFD Society of Canada, Montreal, 2000, pp. 113-118.

[5] P.N. Brown, AND Y. SAAD, Hybrid Krylov methods for nonlinear systems of equations, SIAM J. Sci. Statist. Comput., 11 (1990), pp. 450-481.

[6] R.F. Chen And Z.J. WANG, Fast block lower-upper symmetric Gauß-Seidel scheme for arbitrary grids, AIAA J., 38 (2000), pp. 2238-2245.

[7] W. HackBusch, Iterative Solution of Large Sparse Systems, Springer-Verlag, Berlin, 1994.

[8] M. Eiermann, Fields of values and iterative methods, Linear Algebra Appl., 180 (1993), pp. 167-197.

[9] S.C. Eisenstat, H.C. Elman, And M.H. Schultz, Variational iterative methods for nonsymmetric systems of linear equations, SIAM J. Numer. Anal., 20 (1983), pp. 345-357.

[10] A. Greenbaum, Iterative Methods for Solving Linear Systems, Frontiers Appl. Math. 17, SIAM, Philadelphia, 1997.

[11] C. HiRsch, Numerical Computation of Internal and External Flows: Computational Methods for Inviscid and Viscous Flows, Vol. 2, Wiley, Chichester, UK, 1988.

[12] A. JAmeson, Solution of the Euler equations for two-dimensional transonic flow by a multigrid method, Appl. Math. Comp., 13 (1983), pp. 327-356.

[13] A. JAMESON AND D.A. CAughey, How many steps are required to solve the Euler equations of steady, compressible flow: In search of a fast solution algorithm, AIAA paper 2001-2673, in Proceedings of the 15th AIAA Computational Fluid Dynamics Conference, Anaheim, CA, 2001.

[14] D.A. Knoll, P. McHugh, And D. Keyes, Newton-Krylov methods for low Mach number compressible combustion, AIAA J., 34 (1996), pp. 961-967.

[15] D.A. Knoll And W.J. RIDER, A multigrid preconditioned Newton-Krylov method, SIAM J. Sci. Comput., 21 (1999), pp. 691-710.

[16] B. Koren, Defect correction and multigrid for an efficient and accurate computation of airfoil flows, J. Comput. Phys., 183 (1988), pp. 193-206.

[17] A. MeIster, Comparison of different Krylov subspace methods embedded in an implicit finite volume scheme for the computation of viscous and inviscid flow fields on unstructured grids, J. Comput. Phys., 140 (1998), pp. 311-345.

[18] A. Meister and Th. SonAr, Finite-volume schemes for compressible fluid flow, Surveys Math. Indust., 8 (1998), pp. 1-36.

[19] A. MEISTER AND C. VÖMEL, Efficient preconditioning of linear systems arising from the discretization of hyperbolic conservation laws, Adv. Comput. Math., 14 (2001), pp. 49-73.

[20] N.A. Pierce AND M.B. Giles, Preconditioned multigrid methods for compressible flow computations on stretched meshes, J. Comput. Phys., 136 (1997), pp. 425-445.

[21] A.H. Shapiro, The Dynamics and Thermodynamics of Compressible Fluid Flow, Ronald Press, New York, 1953.

[22] G. StARKe, Field-of-values analysis of preconditioned iterative methods for nonsymmetric elliptic problems, Numer. Math., 78 (1997), pp. 103-117.

[23] R.S. VArga, Matrix Iterative Analysis, Prentice-Hall, Englewood Cliffs, NJ, 1962.

[24] V. Venkatakrishnan and D.J. Mavripilis, Implicit solvers for unstructured meshes, J. Comput. Phys., 105 (1993), pp. 83-91.

[25] V. Venkatakrishnan And D.J. MAVRIPILIS, Implicit method for the computation of unsteady flows on unstructured grids, J. Comput. Phys., 127 (1996), pp. 380-397.

[26] V. VENKATAKRIShNAN, Implicit schemes and parallel computing in unstructured grid CFD, ICASE report 95-28, ICASE, Langley Research Center, Hampton, VA, 1995.

[27] P. Wesseling, An Introduction to Multigrid Methods, Wiley, Chichester, UK, 1992.

[28] P. Wesseling, Principles of Computational Fluid Dynamics, Springer-Verlag, Berlin, 2001. 